\title{
DESCRIPCIÓN EN ÉPOCA SECA DEL MICROHÁBITAT DEL RENACUAJO DE Telmatobius macrostomus (Peters, 1873) "RANA GIGANTE DEL LAGO JUNÍN" EN LOS AFLUENTES DEL LAGO CHINCHAYCOCHA / PERÚ
}

\section{DESCRIPTION IN DRY SEASON OF THE MICROHABITAT OF THE TADPOLE OF Telmatobius macrostomus (Peters, 1873) "LAKE JUNIN FROG" IN THE TRIBUTARIES OF LAKE CHINCHAYCOCHA / PERU}

\author{
Luis Castillo ${ }^{1,2,3,4}$ y Roberto Elias ${ }^{2,5}$
}

\begin{abstract}
Resumen
Telmatobius macrostomus (Peters, 1873) es una especie endémica de ríos y lagunas altoandinas en el centro de Junín y Pasco / Perú, categorizada en peligro de extinción. El presente estudio describe el microhábitat de los renacuajos de T. macrostomus durante la época seca, para ello se evaluaron 10 parámetros fisicoquímicos en subestaciones $\left(\approx 100 \mathrm{~m}^{2}\right)$ y seis en cuadrantes $\left(1 \mathrm{~m}^{2}\right)$. Por medio de una prueba no paramétrica Kruskal-Wallis se determinó que ninguno de los parámetros evaluados en las subestaciones con presencia $(n=10)$ y ausencia $(n=17)$ de renacuajos de T. macrostomus fueron significativamente diferentes (p-value > 0.05); de igual manera los cuadrantes con presencia $(n=29)$ y ausencia $(n=1108)$ no fueron significativamente diferentes $(p-$ value > 0.05). Sin embargo, el análisis de escalamiento no-métrico y un análisis de similaridad (ANOSIM) resultó en una diferencia significativa entre los cuadrantes con presencia y ausencia en una sola subestación $(\mathrm{R}=0.50, \mathrm{p}=0.0004)$. Se establece que los renacuajos de T. macrostomus viven en alta vegetación acuática, cerca de las orillas de los ríos, en sustrato tipo limo, asociados a la presencia de peces nativos del género Orestias y una alta diversidad de diatomeas. Basados en los parámetros evaluados, se concluye que los renacuajos de T. macrostomus son generalistas y que algún tipo de selección de microhábitat en las subestaciones podría estar relacionada a la alteración del hábitat o factores alimenticios, pero en cuadrantes existen características observables asociadas a su presencia. Finalmente, se plantean esfuerzos de conservación a corto plazo para esta especie. Palabras clave: En peligro, Lago Junín, microhábitat, renacuajos, Telmatobius macrostomus.
\end{abstract}

\begin{abstract}
Telmatobius macrostomus (Peters, 1873) is an endemic species of high Andean rivers and lakes in the center of Junin and Pasco / Peru, categorized as endangered. The present study describes the microhabitat of T. macrostomus tadpoles during the dry season, for this, 10 physicochemical parameters were evaluated in substations $(\approx 100 \mathrm{~m} 2)$ and six in quadrants $\left(1 \mathrm{~m}^{2}\right)$. By means of a non-parametric Kruskal-Wallis test it was determined that none of the parameters evaluated in the substations with the presence $(\mathrm{n}=10)$ and absence $(\mathrm{n}=17)$ of tadpoles of T. macrostomus were not different ( $p$ value $>0.05)$, likewise the quadrants with presence $(n=29)$ and absence $(n=1108)$ were not significantly different ( $\mathrm{p}$ value $>0.05)$. However, the non-metric scaling analysis and a similarity analysis (ANOSIM) resulted in a significant difference between the quadrants with presence and absence in a single substation $(R=0.50, p=0.0004)$. It is established that tadpoles of T. macrostomus live in high aquatic vegetation, near the banks of rivers, in silt-type substrate, minimum depth of $30 \mathrm{~cm}$, associated with the presence of native fish of the genus Orestias and a high diversity of diatoms. Based on the evaluated parameters, it is concluded that T. macrostomus tadpoles are generalists and that some type of microhabitat selection in substations could be related to habitat alteration or feeding factors, but there are characteristics in quadrants observables associated with their presence. Finally, short-term conservation efforts are planned for this species. Key words: Endangered, Lake Junin, microhabitat, tadpoles, Telmatobius macrostomus.
\end{abstract}

\section{Introducción}

El lago Chinchaycocha (también conocido como Lago Junín) es el segundo lago más grande del Perú, albergando una alta diversidad de especies y endemismos (SERNANP, 2020); sin embargo, este ecosistema ha sido declarado en emergencia desde el año 2002, por lo que se ha implementado un Plan de Manejo Ambiental para su protección (MINAM, 2017).
La situación actual de los anfibios del mundo agrupa más del $40 \%$ de especies en alguna categoría de amenaza (UICN, 2020); es así, que las especies amenazadas constituyen el principal enfoque de estrategias para su protección (USFWS, 2005). Telmatobius macrostomus (Peters, 1873) conocida como la rana gigante del lago Junín, es una especie que se encuentra actualmente en peligro de extinción (UICN, 2016; SERFOR, 2018) debido a la presencia de 
amenazas como la degradación de su hábitat, contaminación, sobreexplotación y la presencia de especies exóticas invasoras (Angulo, 2008; Aguilar et al., 2010).

Existen pocos estudios sobre los renacuajos de T. macrostomus, se han publicado trabajos en ecología (Guevara \& Morales, 1991; Manyari \& Iannacone, 2006) y taxonomía (Sinsch, 1986; Aguilar \& Valencia 2009), a pesar de su importancia como parte de la cadena trófica en la cuenca del lago Chinchaycocha o como recurso económico y alimenticio (Sinsch, 1986; Watson et al., 2017a).

Telmatobius macrostomus es una especie estrictamente acuática (Vellard, 1951), endémica de Perú y se distribuye por las regiones de Junín y Pasco. Se le puede encontrar en pequeñas lagunas y riachuelos, pero también en el río Mantaro (valle superior) y el lago Junín, con una distribución altitudinal que va desde los $3400 \mathrm{msnm}$ hasta los $4660 \mathrm{msnm}$ (Sinsch, 1986).

Los renacuajos de T. macrostomus, alcanzan medidas de hasta $202 \mathrm{~mm}$ de longitud total, este gran tamaño es un ejemplo del fenómeno conocido como gigantismo larval (Vellard, 1951). Se conoce que renacuajos, metamorfos, juveniles y adultos coexisten en todas las épocas del año, debido a su extenso desarrollo larvario y actividad reproductiva constante, posiblemente vinculada con la temperatura estable del agua (Vellard, 1951; Sinsch, 1986; Watson et al., 2017b); por esta razón, es importante conocer las variables ambientales que afectan el comportamiento individual, a esto se le conoce como microhábitat (Morris, 1987). Este estudio describe el microhábitat del renacuajo de T. macrostomus en el ecosistema de afluentes del lago Chinchaycocha y enfatiza esfuerzos de conservación inmediatos.

\section{Materiales y métodos}

$\underline{\text { Área de estudio }}$

El estudio fue desarrollado en la cuenca del lago Chinchaycocha, en la Región Junín, a una elevación de aproximadamente 4100 msnm (Figura 1). Fueron examinadas ocho estaciones: siete afluentes del lago (E1-E7) y un canal artificial (E8), distribuidos en la Reserva Nacional de Junín (RNJ) y su zona de amortiguamiento, en los distritos de Carhuamayo, Ondores y Junín (Tabla 1). El clima fue característico de la puna altoandina, con una temperatura ambiental muy variada durante el día (6 a $27{ }^{\circ} \mathrm{C}$ ), humedad relativa $(30-79 \%)$ e irradiancia solar $(0.36$ - 239 $\mathrm{mW} / \mathrm{m} 2$ ). El área de estudio está afectada por la ganadería intensiva, agricultura y aguas residuales de comunidades aledañas (Tabla 1).

\section{Diseño de muestreo}

Las ocho estaciones fueron divididas en 27 subestaciones (unidad muestral 1) seleccionadas al azar. Cada subestación tuvo $50 \mathrm{~m}$ de longitud y estuvieron separadas por un mínimo de $300 \mathrm{~m}$ una de otra para mantener su independencia (Rueda et al.,
2006). Cuatro estaciones (E1, E2, E3 y E4) fueron seleccionadas porque existía un registro de presencia de renacuajos (Watson et al., 2017a b), mientras que en las estaciones E5, E6, E7 y E8 se evaluaron una o dos subestaciones por considerarse lugares probables con presencia de renacuajos (según información de pobladores locales).

En cada subestación se establecieron parcelas de 1 x $1 \mathrm{~m}$, denominadas cuadrantes (unidad muestral 2), también seleccionadas al azar y consideradas para caracterizar y comparar a menor escala el microhábitat (Rueda-Solano et al., 2015). La cantidad de cuadrantes dependió proporcionalmente del ancho de las subestaciones.

\section{Colecta biológica}

La evaluación se realizó de 09:00 a 17:00 h entre el 11 y 22 de julio del 2016 en época seca; no se realizó la evaluación en época húmeda por problemas logísticos. Los renacuajos fueron capturados con redes cuchara “dip-net" con un desplazamiento en dirección río-arriba - "contracorriente", examinando cuidadosamente todos los espacios disponibles, como lo indica Watson et al. (2017b); luego la especie fue identificada en campo usando su descripción y claves taxonómicas (Peters, 1873; Sinsch, 1986; Aguilar \& Valencia, 2009) y fue agrupada como renacuajo usando la tabla de Gosner (1960).

La evaluación de cada estación en campo se llevó a cabo por uno o dos evaluadores, durante dos horas aproximadamente. En la búsqueda también se registró la presencia de peces del género Orestias (Valenciennes, 1839), y de las especies Trichomycterus oroyae (Eigenmann \& Eigenmann, 1889) y Oncorhynchus mykiss (Walbaum, 1792).

Las plantas acuáticas encontradas junto a los renacuajos fueron colectadas y depositadas en frascos de $200 \mathrm{ml}$ con formol al 7\%, para la posterior identificación del perifiton asociado. Los organismos identificados fueron agrupados por phyla, en el Laboratorio de Ecología Acuática de la Facultad de Ciencias Biológicas de la Universidad Nacional Mayor de San Marcos.

Evaluación de parámetros en las subestaciones

En cada subestación se evaluaron 10 parámetros físicoquímicos como temperatura del agua, $\mathrm{pH}$, sólidos disueltos totales (TDS por sus siglas en inglés), conductividad, salinidad, los cuales fueron medidos con un multiparámetro marca Extech PH100 a $50 \mathrm{~cm}$ de la orilla aproximadamente; el porcentaje de oxígeno saturado (\% OS) fue medido con el equipo EZODO 7031, el porcentaje de cobertura superficial, porcentaje de vegetación, profundidad promedio y finalmente se calculó el ancho promedio del río con tres medidas, al inicio, medio y final de la subestación.

\section{Evaluación de parámetros en los cuadrantes}

Para cada cuadrante se evaluaron seis parámetros. Primero, se estimó el porcentaje (múltiplo de 5) de cobertura superficial y vegetación sumergida. Segundo, 
se calculó la distancia aproximada a la orilla $(0,1,2 \mathrm{~m}$ a más), la profundidad, el tipo de sustrato: arcilla, limo, arena, grava o piedra (Wolman, 1954) y el nivel estimado de la corriente de agua (baja, media o alta). Tratamiento de datos

El método usual para determinar la selección de microhábitat es mediante la correlación de variables que muestran una potencial influencia en la selección de microhábitat (Bozeman \& Grossman, 2019).

No hubo normalidad en los parámetros, por lo que se usó la prueba no paramétrica de Kruskal-Wallis para comparar las subestaciones y los cuadrantes con y sin presencia de renacuajos. Solo para la subestación E3S2, se desarrolló un análisis escalamiento multidimensional no-métrico debido a la heterogeneidad de sus variables categóricas, comparándose las variables cuantitativas no transformadas (\% cobertura superficial, \% vegetación sumergida y profundidad) usando la prueba no paramétrica de Kruskal-Wallis.

El resultado fue contrastado con un análisis de similaridad (ANOSIM) basado en 10000 permutaciones al azar de la matriz de disimilaridad.

Luego, se determinaron los valores mínimos (min), máximos (máx) y promedios de los parámetros fisicoquímicos del microhábitat de las subestaciones. Los análisis fueron ejecutados en PAST 3.15 (Hammer et al., 2001). La vegetación sumergida, cobertura superficial y profundidad fueron analizadas como promedios en las subestaciones.

Los parámetros de los cuadrantes con renacuajos como distancia aproximada a la orilla, tipo de sustrato, nivel estimado de la corriente de agua y presencia de los potenciales competidores acuáticos (peces de Orestias, Trichomycterus oroyae) y depredadores (Oncorhynchus mykiss), fueron representados por frecuencias de ocurrencia (Watson et al., 2017a). El perifiton fue identificado hasta especie y agrupados por phyla usando el AlgaeBase (Guiry \& Guiry, 2017).

\section{Resultados}

\section{Distribución y abundancia}

De las ocho estaciones evaluadas, seis estuvieron ocupadas por un total de 34 renacuajos. La cantidad de renacuajos reportados por subestación está representada en la Tabla 1. También se observó, dentro de una estación con cinco o más subestaciones, que los renacuajos de T.macrostomus no se encontraron distribuidos en todas (Figura 1).

Un total de 1137 cuadrantes fueron evaluados y de 29 de ellos se registró la presencia de renacuajos de T. macrostomus (cinco cuadrantes con dos renacuajos cada uno). La estación E2 presentó la mayor abundancia $(n=13)$ en tres subestaciones, seguida de la estación E3 $(n=10)$ en solo una subestación. Las E1 y E4 fueron las únicas que registraron la presencia de cinco metamorfos en total, junto a renacuajos. En la subestación E1S3, los renacuajos se encontraron junto a un individuo juvenil de T. macrostomus. Mientras, a unos metros de la subestación E3S5, se capturó un adulto.

Descripción del microhábitat

Los diez parámetros evaluados entre las subestaciones con presencia y ausencia de renacuajos no mostraron diferencias significativas ( $\mathrm{p}>0.05$, Kruskal Wallis). El análisis univariado de algunos parámetros para cada subestación reveló que la temperatura del agua, cobertura superficial, vegetación sumergida, la profundidad y el ancho fueron las variables que presentaron un coeficiente de variación mayor a 30 (Tabla 2). La vegetación que predomina en las riberas son pastizales, césped de puna y plantas herbáceas. Las subestaciones con o sin renacuajos fueron caracterizadas por un alto porcentaje de vegetación sumergida y cobertura superficial (Tabla 2). El pH presentó el valor mínimo (7.67) y máximo (10.02) reportados; igualmente, la profundidad (23.4 $\mathrm{cm})$ fue la menor de todas.

La mayor abundancia de renacuajos se dio en la subestación E3S2, lo cual permitió obtener un contraste del análisis de escalamiento multidimensional no paramétrico, encontrándose diferencias significativas entre cuadrantes con y sin renacuajos (Stress $=0.18$, ANOSIM R $=0.50, p=0.0004)$. Las variables que permitieron esta diferencia en E3S2 fueron los promedios del porcentaje de cobertura superficial (presencia: media $=49 \%, \min =20 \%$, máx $=80 \%$; ausencia: media $=25 \%$, $\min =0 \%$, máx $=80 \%$; -value $=0.03$ ), vegetación sumergida (presencia: media $=$ $74 \%, \min =20 \%$, máx $=80 \%$; ausencia: media $=41 \%$, $\min =0 \%$, máx $=100 \% ; \mathrm{p}$-value $=0.04)$ y la profundidad (presencia: media $=40 \mathrm{~cm}, \min =30 \mathrm{~cm}$, máx $=45 \mathrm{~cm}$; ausencia $=32 \mathrm{~cm}, \min =25 \mathrm{~cm}$, máx $=$ $40 \mathrm{~cm}$; p-value $=0.003$ ) de los cuadrantes.

Los parámetros numéricos de los cuadrantes con presencia y ausencia de renacuajos no mostraron diferencias significativas ( $\mathrm{p}>0.05$, Kruskal Wallis). Los 34 renacuajos de T. macrostomus fueron encontrados a menos de un metro de la orilla $(100 \%, \mathrm{n}$ $=29)$, en sustrato tipo limo $(93 \%, \mathrm{n}=27)$, arcilla $(3.5 \%$, $\mathrm{n}=1)$, grava $(3.5 \%, \mathrm{n}=1)$, corriente baja $(100 \%, \mathrm{n}=$ 29), también en áreas con presencia de vegetación acuática sumergida como Myriophyllum sp. "cola de zorro", emergentes como Hydrocotyle sp. "sombrero de sapo" y Scirpus sp. "totora". También estaban presentes junto a peces del género Orestias $(93 \%, \mathrm{n}=$ 27) y una alta diversidad de diatomeas - phylum Bacillariophyta (Figura 2, Tabla 3). Los renacuajos no fueron encontrados con T. oroyae $(0 \%, \mathrm{n}=29) \mathrm{u}$ $O$. mykiss en ninguna de las estaciones evaluadas.

\section{Discusión \\ Distribución y abundancia}

Desde 1998 se reportan subpoblaciones de renacuajos de T. macrostomus en riachuelos alrededor del lago Junín (Camacho, 2001; Loza \& Mendoza, 
2017; Watson et al., 2017a b). En este estudio se confirma la presencia de renacuajos en las estaciones E1, E2, E3 y E4 (Figura 1) similar a lo registrado por Watson et al. (2017a) en los meses de octubre del 2015 y abril del 2016. Así mismo, en la E5, según guardaparques de la RNJ e investigadores, se confirma la presencia de renacuajos después de 7 años. La E6 tiene el registro más nororiental de la zona de amortiguamiento de la RNJ y se localiza en zona de exploración minera, según pobladores locales.

Se asume que las subpoblaciones de los renacuajos de T. macrostomus están severamente fragmentadas entre subestaciones debido a la ganadería, remoción de vegetación riparia y modificación de los cauces, lo que habría afectado su distribución y actividad natural.

Descripción del microhábitat

La preferencia por lugares con alto porcentaje de macrófitas acuáticas guarda relación con su fuente de alimento de tipo perifítico (Guevara \& Morales, 1991), esto se comprueba por la asociación a una alta diversidad de diatomeas en los cuadrantes con renacuajos; y de macroinvertebrados (Watson et al., 2017b). También, la vegetación acuática les brinda protección contra depredadores naturales, como aves.

Se asume que los renacuajos de T. macrostomus, durante el día están posados o alimentándose mayormente de diatomeas y ocultos entre las plantas acuáticas ribereñas. Desconocemos su actividad nocturna, probablemente se encuentren activos desplazándose a lo largo de los riachuelos.

El limo fue el sustrato predominante para T. macrostomus, similar a lo reportado por Watson et al. (2017b), sin embargo, este tipo de sustrato es común en zonas afectadas por malas prácticas (SEPA, 2010) probablemente relacionadas con el embalse del lago Chinchaycocha desde 1932 (Robdell et al., 2014). También, proveen las condiciones necesarias para el desarrollo de individuos de la familia Chironomidae (Dudgeon, 1994), considerados indicadores de pobre calidad de agua y asociados negativamente a la presencia de T. macrostomus (Watson et al., 2017b).

La alta variación de la temperatura del agua está relacionada con la hora del día en el área de estudio; la baja temperatura ocurre en la noche y la alta temperatura durante el día (datos no publicados), así que los renacuajos deben estar adaptados a estos cambios, pero no se conoce alguna relación. Acerca de los parámetros físicoquímicos, el valor mínimo de vegetación sumergida y el porcentaje de cobertura superficial (21.7\% y 40\%, respectivamente) indican un microhábitat débil muy similar a la subestación E3S5 con $7.6 \%$ (sin renacuajos). Los anchos de los riachuelos no tienen relación con los renacuajos porque fueron encontrados en casi todas las medidas con excepción a 10 y 10.5 metros. Por otro lado, la profundidad mínima de cuadrantes con renacuajos fue de $27 \mathrm{~cm}$, por ello, consideramos este valor como mínimo para su microhábitat.
Valores menores a 6.5 y mayores a $10 \mathrm{de} \mathrm{pH}$ alteran el desarrollo de anfibios (Padhye \& Ghate, 1988; Pierce, 1985, 1993). Estos valores anómalos indican contaminación de origen minero, como los vertidos en la zona norte del lago Junín, con reportes de entre 2.8 a 6.2 (Camacho, 2001), distantes a las localidades aquí evaluadas, pero cuyos metales ocasionarían una alta mortalidad (Iannacone \& Manyari, 2003). En este trabajo, la subestación E8S1 (canal artificial sin circulación de agua) presentó el valor máximo de $\mathrm{pH}$ (10.02) que afecta negativamente a los anfibios, representando un mal hábitat para los renacuajos de T. macrostomus. Watson et al. (2017b) registraron un valor promedio de 8.38 para los sitios con T. macrostomus, muy similares a los nuestros; por esta razón, sugerimos un rango óptimo del $\mathrm{pH}$ entre 8.3 y 8.4 para los renacuajos de T. macrostomus.

Medidas de conservación

Las estaciones E1, E2 y E3 tienen la mayor cantidad de renacuajos, presencia de metamorfos y adultos, además de adecuados parámetros fisicoquímicos y biológicos (altos porcentajes de vegetación sumergida y superficial) indicando un mayor número de microhábitats para desarrollar esfuerzos de conservación in situ.

En su área de distribución se llevan a cabo diferentes amenazas en todos los afluentes, como la limpieza de riachuelos o canales, donde se extrae la vegetación riparia para aumentar la irrigación y evitar la inundación de áreas de pastoreo, ocasionando la destrucción del hábitat y la extracción de la especie. Por ejemplo, en la subestación E3S1, se notó una poca o nula presencia de vegetación ribereña y ningún renacuajo fue encontrado, aunque a $300 \mathrm{~m}$ en la E3S2 se registró la mayor abundancia de renacuajos. También la ganadería, en zonas con presencia de T. macrostomus, altera la estructura de los riachuelos (remoción de vegetación acuática al alimentarse) y la composición química del agua (orina y heces). Ante ambos casos son necesarias medidas de ordenamiento territorial para proteger el hábitat de la especie (Grant et al., 2016) y mantener poblaciones ecológicamente viables de T. macrostomus.

Estas evidencias enfatizan la necesidad de la ejecución de un plan de conservación de la Rana Gigante del Lago Junín, T. macrostomus, el cual está siendo implementado por la Fundación Zoológica de Denver, GRUPO RANA y varios aliados nacionales e internacionales a través de formas estructuradas de investigación, educación y manejo ambiental (Watson et al., 2016).

\section{Conclusiones}

Los renacuajos de T.macrostomus están relacionados a un microhábitat caracterizado por un sustrato tipo limo, corrientes bajas, asociación a la orilla, presencia de peces del género Orestias, alta diversidad de diatomeas, a profundidades de más de 27 
cm, con vegetación sumergida y superficial generalmente alta. En base a los parámetros evaluados en las subestaciones, los renacuajos de T. macrostomus son considerados generalistas y su preferencia por un microhábitat podría estar delimitado por factores alimenticios, relaciones interespecíficas o por la alteración de su hábitat.

\section{Agradecimientos}

Agradecemos al Denver Zoological Foundation por el financiamiento total del proyecto. Al curador César Aguilar e Iris Samanez, del Departamento de Herpetología y Departamento de Limnología del Museo de Historia Natural Javier Prado de la UNMSM, por las primeras revisiones del manuscrito; al excurador Jesús Córdova por sus recomendaciones y brindarme un espacio de investigación. A Andrew Watson, Austin Fitzgerald, Pilar Valentín, Maura Fernández, guardaparques voluntarios de la RNJ y voluntarios del Cuerpo de Paz por su apoyo en campo. A Oscar Damián-Baldeón y Rogger Moreno por la elaboración inicial del mapa. A Karen Roa por la identificación del perifiton. Al Servicio Nacional de Áreas Naturales Protegidas y al Servicio Forestal y de Fauna Silvestre por otorgar los permisos de investigación (RJ N005-2016-SERNANP-RNJ-JEF y RDG N ${ }^{\circ}$ 285-2016-SERFOR/DGGDPFFS).

\section{Literatura citada}

Aguilar C. \& Valencia N. 2009. Relaciones filogenéticas entre telmatobiinidos (Anura, Ceratophryidae, Telmatobiinae) de los Andes centrales basado en la morfología de los estados larval y adultos. Revista Peruana de Biología, 16(1): 43-50.

DOI: $10.15381 / \mathrm{rpb} . v 16 i 1.174$

Aguilar C., Ramírez C., Rivera D., Siu-Ting K., Suarez J. \& Torres C. 2010. Anfibios andinos del Perú fuera de Áreas Naturales Protegidas: amenazas y estado de conservación. Revista Peruana de Biología, 17(1): 5-28. http://www.scielo.org.pe/pdf/rpb/v17n1/a02v17n1.pdf.

Angulo A. 2008. Conservation Needs of Batrachophrynus and Telmatobius Frogs of the Andes of Peru. Conservation and Society, 6(4): 328-333. DOI: $10.4103 / 0972-4923.49196$.

Bozeman B. \& Grossman G. 2019. Foraging behaviour and optimal microhabitat selection in Yukon River Basin nonanadromous Dolly Varden Charr (Salvelinus malma). Ecology of freshwater fish, 28(4): 586-601. DOI: 10.1111/eff.12477.

Camacho R. 2001. Propuesta de Manejo para la Rana de Junín (Batrachophrynus macrostomus). Tesis para optar el grado de Magister Scientiae. Universidad Nacional Agraria La Molina. Lima, Perú.

Dudgeon D. 1994. The functional significance of selection of particles by aquatic animals during building behavior. 289-312. In: Wotton R.S. (Ed) The biology of particles in aquatic systems. Lewis Publishers.

Gosner K. 1960. A Simplified Table for Staging Anuran Embryos and Larvae with Notes on Identification. Herpetologica, 16(3): 183-190. https://www.jstor.org/stable/3890061.
Grant E., Miller D., Schmidt B., Adams M., Amburgey S., Chambert T., Cruickshank S., Fisher R., Green D., Hossack B., Johnson P., Joseph M., Rittenhouse T., Ryan M., Waddle H., Walls S., Bailey L., Fellers G., Gorman T., Ray A., Pilliod D., Price S., Saenz D., Sadinski W. \& Muths E. 2016. Quantitative evidence for the effects of multiple drivers on continental-scale amphibian declines. Scientific Report, 6: 25625. DOI: 10.1038/srep25625.

Guevara M. \& Morales V. 1991. Nota sobre la composición algal de la dieta en larvas de Batrachophrynus Peters. 1873 (Anura: Leptodactylidae) del Perú. Boletín del Museo de Historia Natural "Javier Prado", 41: 6-7.

Guiry M. \& Guiry G. 2017. AlgaeBase. World-wide electronic publication. National University of Ireland, Galway. Consultado el 17 de julio de 2017 de: http://www.algaebase.org.

Hammer O., Harper D. \& Ryan P. 2001. PAST: Paleontological Statistics Software Package for Education and Data Analysis. Palaeontologia Electronica, 4(1): 1-9. https://palaeo-electronica.org/2001_1/past/past.pdf.

Iannacone J. \& Manyari G. 2003. Ecotoxicidad del mercurio, cadmio y plomo sobre renacuajos de la rana de Junín Batrachophrynus macrostomus Peters 1873 (Anura: Leptodactylidae). Revista Brasileira de Toxicología, 16(1): 15-20. https://www.researchgate.net/profile/JoseIannacone/publication/270277201_Ecotoxicity_of_merc ury_cadmium_and_lead_on_Lake_Junin_Giant_Frog_B atrachophrynus_macrostomus_Peters_1873_Anura_Lept odactylidae_tadpoles/links/54a55eb90cf257a63608cc3b/ Ecotoxicity-of-mercury-cadmium-and-lead-on-LakeJunin-Giant-Frog-Batrachophrynus-macrostomusPeters-1873-Anura-Leptodactylidae-tadpoles.pdf.

Loza A. \& Mendoza W. 2017. Evaluación poblacional y estado de conservación de Telmatobius macrostomus Peters, 1873 (Anura: Telmatobiidae) en humedales altoandinos, Región Pasco-Perú. Revista de Investigaciones Altoandinas, 19(2): 145-156.

DOI: $10.18271 /$ ria.2017.273.

Manyari G. \& Iannacone J. 2006. Relación peso-longitud en larvas de la Rana de Junin Batrachophrynus macrostomus Peters 1873 (Anura: Leptodactylidae) para su uso en ecotoxicología en condiciones de zoocriadero. Biologist (Lima), 4(2): 1415 .

http://revistas.unfv.edu.pe/index.php/rtb/article/view/56 5.

MINAM (Ministerio del Ambiente). 2017. Resolución Suprema N ${ }^{\circ}$ 005-2017-MINAM. Aprueban el Plan de Manejo Ambiental sostenible Chinchaycocha 2017-2021. El Peruano (Normas Legales), Sábado 28 de octubre de 2017, XXXIV(14264): 9-9. 1581502-5.

https://www.minam.gob.pe/wpcontent/uploads/2017/10/RS_005-2017-MINAM.pdf.

Morris D. 1987. Ecological scale and habitat use. Ecology, 68(2): 362-369. DOI: 10.2307/1939267.

Padhye A. \& Ghate H. 1988. Effect of altered pH on embryos and tadpoles of the frog Microhyla ornata. The Herpetological Journal, 1(7): 276-279.

https://www.thebhs.org/publications/the-herpetologicaljournal/volume-1-number-7-december-1988/1188-05effect-of-altered-ph-on-embryos-and-tadpoles-of-thefrog-microhyla-ornata. 
Peters W. 1873. Über zwei Gisftschlangen aus Afrika und über neue oder weniger bekannte Gattungen und Arten von Batrachiern, 1873: 411-414.

Pierce B. 1985. Acid tolerance in amphibians. BioScience, 35(4): 239-243.

Pierce B. 1993. The effects of acid precipitation on amphibians. Ecotoxicology, 2: 65-77.

Robdell D., Delman E., Abbott M., Besonen M. \& Tapia P. 2014. The heavy metal contamination of Lake Junín National Reserve, Peru: An unintended consequence of the juxtaposition of hydroelectricity and mining. GSA TODAY, 24(8): 4-10. DOI: 10.1130/GSATG200A.1.

Rueda J., Castro F. \& Cortez C. 2006. Técnicas para el inventario y muestreo de anfibios: Una compilación. 135172. En: Angulo A., Rueda-Almonacid J., RodriguezMahecha J., La Marca E. Técnicas de inventario y monitoreo para los anfibios de la región tropical andina. Conservación Internacional. Serie Manuales de Campo $\mathrm{N}^{\circ}$ 2. Bogotá (Colombia).

Rueda-Solano L., Vargas F. \& Rivera M. 2015. The highland tadpole of the harlequin frog Atelopus carrikeri (Anura: Bufonidae) with an analysis of its microhabitat preference. Salamandra, 51(1): 25-32.

SEPA (Scottish Environment Protection Agency). 2010. Engineering in the water environment: good practice guide. Sediment management. 1st ed. Scotland. Engineering guidance. https://www.sepa.org.uk/media/151049/wat-sg-26.pdf.

SERFOR (Servicio Nacional Forestal y de Fauna Silvestre). 2018. Libro Rojo de la Fauna Silvestre Amenazada del Perú. Primera edición. Serfor. Lima, Perú. https://sinia.minam.gob.pe/documentos/libro-rojo-faunasilvestre-amenazada-peru.

SERNANP (Servicio Nacional de Áreas Naturales Protegidas por el Estado). 2020. Reserva Nacional de Junín. Un espejo en medio de los Andes. Primera Edición. Perú.

Sinsch U. 1986. Anfibios de la sierra central del Perú. Una clave de identificación para adultos y larvas. Boletín de Lima, (45): 23-33. https://www.boletindelima.com/198645.htm.
UICN (Unión Internacional para la Conservación de la Naturaleza). 2016. IUCN Red List of Threatened Species. Version 2015. 4. Consultado el 29 de Mayo de 2016 de: http://www.iucnredlist.org.

UICN (Unión Internacional para la Conservación de la Naturaleza). 2020. La Lista Roja de Especies Amenazadas de la UICN. Versión 2020-1. https://www.iucnredlist.org.

USFWS (United States Fish and Wildlife Service). 2005. Why Save Endangered Species? Endangered Species Program. www.fws.gov/endangered/.

Vellard J. 1951. Estudios sobre batracios andinos. I. El grupo Telmatobius y formas afines. Memorias del Museo de Historia Natural "Javier Prado", 1: 1-89. https://museohn.unmsm.edu.pe/docs/Memorias/Memori a\%20N\%C2\%B0\%201.pdf.

Watson A., Fitzgerald A., Baldeón O., Chamorro A. \& Castillo L. 2016. Ranas Altoandinas en la Región de Junín: Estado Actual y Plan Estratégico de Conservación. Technical Report. DOI: 10.13140/RG.2.2.16588.31365.

Watson A., Fitzgerald A. \& Baldeón O. 2017a. Diet composition and prey selection of Telmatobius macrostomus, the Junín giant frog. Endangered Species Research, 32: 117-121. DOI: $10.3354 / \mathrm{esr} 00785$.

Watson A., Fitzgerald A., Baldeón O. \& Elias R. 2017b. Habitat characterization, occupancy and detection probability of the Endangered and endemic Junín giant frog, Telmatobius macrostomus. Endangered Species Research, 32: 429-436. DOI: 10.3354/esr00821.

Wolman M. 1954. A method of sampling course river-bed material. Eos (Earth \& Space Science News) Transactions, 35(6): 951-956. AGU (American Geophysical Union). https://doi.org/10.1029/TR035i006p00951. 


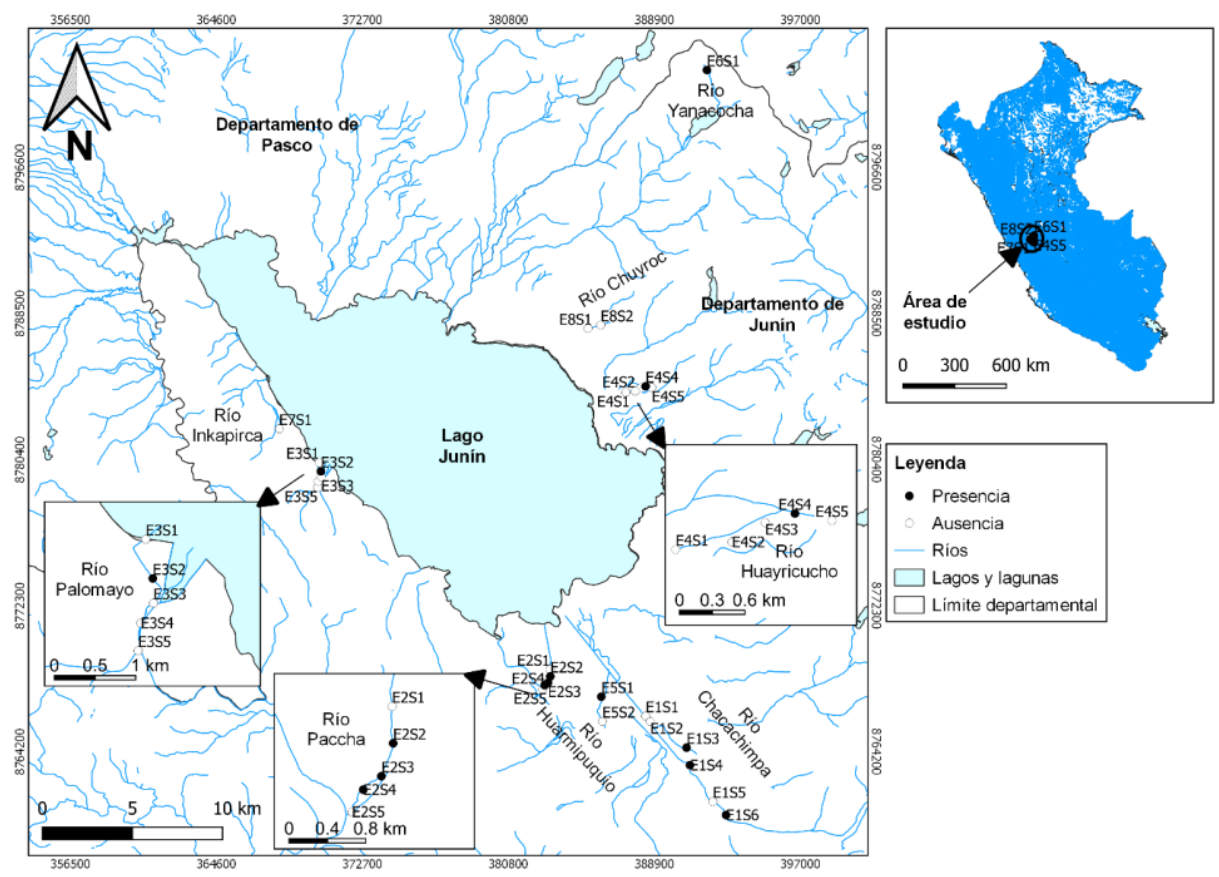

Figura 1. Área de estudio de renacuajos de Telmatobius macrostomus en los afluentes del lago Junín, en los andes centrales del Perú.

Tabla 1. Información detallada de las estaciones, subestaciones, cuadrantes y abundancia.

\begin{tabular}{|c|c|c|c|c|c|c|c|}
\hline 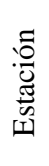 & 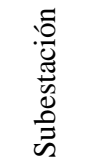 & 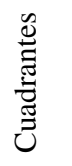 & 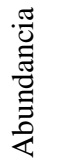 & Latitud & Longitud & $\begin{array}{l}\text { Elevación } \\
\text { (msnm) }\end{array}$ & Descripción \\
\hline \multirow[t]{6}{*}{ E1 } & E1S1 & 100 & 0 & 388413 & 8766180 & 4095 & \multirow{6}{*}{$\begin{array}{l}\text { Chacachimpa. Caudal perenne, afectada por aguas } \\
\text { servidas y ganadería. Ancho de } 5 \text { a } 10 \mathrm{~m} \text {. }\end{array}$} \\
\hline & E1S2 & 100 & 0 & 388666 & 8765872 & 4096 & \\
\hline & E1S3 & 100 & 3 & 390676 & 8764428 & 4101 & \\
\hline & E1S4 & 81 & 1 & 390865 & 8763456 & 4103 & \\
\hline & E1S5 & 60 & 0 & 392137 & 8761436 & 4119 & \\
\hline & E1S6 & 32 & 2 & 392865 & 8760692 & 4127 & \\
\hline \multirow[t]{5}{*}{ E2 } & E2S1 & 30 & 0 & 383005 & 8768762 & 4089 & \multirow{5}{*}{$\begin{array}{l}\text { Paccha. Caudal perenne, afectada por ganadería, alta } \\
\text { sedimentación y vías vehiculares. Ancho de } 1 \text { a } 3 \mathrm{~m} \text {. }\end{array}$} \\
\hline & E2S2 & 30 & 2 & 383118 & 8768377 & 4090 & \\
\hline & E2S3 & 30 & 7 & 382996 & 8768028 & 4091 & \\
\hline & E2S4 & 30 & 4 & 382807 & 8767885 & 4092 & \\
\hline & E2S5 & 20 & 0 & 382691 & 8767650 & 4092 & \\
\hline \multirow[t]{5}{*}{ E3 } & E3S1 & 21 & 0 & 370301 & 8780238 & 4085 & \multirow{5}{*}{$\begin{array}{l}\text { Palomayo. Caudal perenne, afectada por ganadería, } \\
\text { lavadero de ropa. Ancho } 1 \text { a } 2 \mathrm{~m} \text {. }\end{array}$} \\
\hline & E3S2 & 23 & 10 & 370390 & 8779751 & 4091 & \\
\hline & E3S3 & 31 & 0 & 370400 & 8779444 & 4095 & \\
\hline & E3S4 & 20 & 0 & 370245 & 8779195 & 4102 & \\
\hline & E3S5 & 16 & 0 & 370214 & 8778850 & 4111 & \\
\hline \multirow[t]{5}{*}{ E4 } & E4S1 & 25 & 0 & 387308 & 8784125 & 4089 & \multirow{5}{*}{$\begin{array}{l}\text { Huayricucho. Caudal perenne, afectada por aguas } \\
\text { servidas, ganadería y agricultura, elaboración de } \\
\text { chuño y vías vehiculares. Ancho de } 1 \text { a } 2 \mathrm{~m} \text {. }\end{array}$} \\
\hline & $\mathrm{E} 4 \mathrm{~S} 2$ & 20 & 0 & 387827 & 8784197 & 4089 & \\
\hline & E4S3 & 21 & 0 & 388132 & 8784378 & 4092 & \\
\hline & E4S4 & 33 & 2 & 388405 & 8784464 & 4097 & \\
\hline & E4S5 & 24 & 0 & 388746 & 8784399 & 4101 & \\
\hline \multirow[t]{2}{*}{ E5 } & E5S1 & 80 & 1 & 385948 & 8767252 & 4093 & \multirow{2}{*}{$\begin{array}{l}\text { Huarmipuquio. Caudal perenne, afectada por } \\
\text { ganadería y alta sedimentación. Ancho de } 7 \text { a } 8 \mathrm{~m} \text {. }\end{array}$} \\
\hline & E5S2 & 80 & 0 & 386002 & 8765885 & 4094 & \\
\hline E6 & E6S1 & 30 & 2 & 391804 & 8802000 & 4335 & $\begin{array}{l}\text { Yanacocha. Caudal perenne, afectada por ganadería y } \\
\text { alta sedimentación. Ancho de } 2.5 \mathrm{~m} \text {. }\end{array}$ \\
\hline E7 & E7S1 & 31 & 0 & 368082 & 8782108 & 4108 & $\begin{array}{l}\text { Inkapirca. Caudal perenne, afectada por lavadero de } \\
\text { ropa. Ancho de } 2 \mathrm{~m} \text {. }\end{array}$ \\
\hline E8 & E8S1 & 30 & 0 & 385208 & 8787674 & 4085 & $\begin{array}{l}\text { Chuyroc. Canal artificial, agua empozada proveniente } \\
\text { del lago, afectada por residuos sólidos. Ancho de } 2.5 \\
\text { a } 4 \text { m. }\end{array}$ \\
\hline
\end{tabular}


Enero - Julio 2021

MICROHÁBITAT DEL RENACUAJO DE Telmatobius macrostomus EN JUNÍN / PERÚ

Tabla 2. Análisis univariado de los parámetros evaluados en las subestaciones con renacuajos (en negrita) y sin renacuajos.

\begin{tabular}{lcccc}
\hline Parámetros fisicoquímicos y biológicos & Mínimo & Máximo & Promedio & $\begin{array}{c}\text { Coeficiente de } \\
\text { variación }\end{array}$ \\
\hline Temperatura agua $\left({ }^{\circ} \mathrm{C}\right)$ & $\mathbf{3 . 0 2}$ & $\mathbf{1 9 . 3}$ & $\mathbf{1 2 . 0} \pm \mathbf{4 . 5 9}(\mathbf{n}=\mathbf{1 0})$ & $\mathbf{3 8 . 2 3}$ \\
& 6.3 & 17 & $11.9 \pm 3.38(\mathrm{n}=17)$ & 28.43 \\
TDS $(\mathrm{mg} / \mathrm{l})$ & $\mathbf{1 9 8}$ & $\mathbf{3 4 0}$ & $\mathbf{2 5 6 . 3} \pm \mathbf{4 2 . 8 4}(\mathbf{n}=\mathbf{1 0})$ & $\mathbf{1 6 . 7 1}$ \\
& 179 & 364 & $253.1 \pm 37.81(\mathrm{n}=17)$ & 14.94 \\
Conductividad (uS) & $\mathbf{2 8 9}$ & $\mathbf{4 8 7}$ & $\mathbf{3 6 7 . 9} \pm \mathbf{6 2 . 7 9}(\mathbf{n}=\mathbf{1 0})$ & $\mathbf{1 7 . 0 7}$ \\
& 246 & 517 & $356.82 \pm 56.84(\mathrm{n}=17)$ & 15.93 \\
Salinidad $(\mathrm{ppm})$ & $\mathbf{1 2 9}$ & $\mathbf{2 3 1}$ & $\mathbf{1 7 1 . 9} \pm \mathbf{3 0 . 1 7}(\mathbf{n}=\mathbf{1 0})$ & $\mathbf{1 7 . 5 5}$ \\
& 121 & 250 & $170.5 \pm 26.7(\mathrm{n}=17)$ & 15.68 \\
pH & $\mathbf{7 . 8 1}$ & $\mathbf{8 . 6 6}$ & $\mathbf{8 . 2 8} \pm \mathbf{0 . 2 8}(\mathbf{n}=\mathbf{1 0})$ & $\mathbf{3 . 4 1}$ \\
& 7.67 & 10.02 & $8.48 \pm 37.81(\mathrm{n}=17)$ & 6.89 \\
OD $(\%)$ & $\mathbf{3 4 . 5}$ & $\mathbf{5 5}$ & $\mathbf{4 3 . 6 4} \pm \mathbf{7 . 6 3}(\mathbf{n}=\mathbf{9})$ & $\mathbf{1 7 . 4 9}$ \\
& 18.5 & 53.89 & $41.46 \pm 11.07(\mathrm{n}=17)$ & 26.7 \\
Cobertura superficial $(\%)$ & $\mathbf{2 1 . 7}$ & $\mathbf{8 7 . 8}$ & $\mathbf{6 0 . 9 5} \pm \mathbf{2 2 . 4 6}(\mathbf{n}=\mathbf{1 0})$ & $\mathbf{3 6 . 8 5}$ \\
& 7.62 & 82.38 & $61.61 \pm 17.84(\mathrm{n}=17)$ & 30.42 \\
Vegetación sumergida $(\%)$ & $\mathbf{4 0}$ & $\mathbf{9 5}$ & $\mathbf{6 9 . 8 2} \pm \mathbf{2 1 . 8 5}(\mathbf{n}=\mathbf{1 0})$ & $\mathbf{3 1 . 3}$ \\
Ancho $(\mathrm{m})$ & 7.86 & 99.33 & $70.22 \pm 23.87(\mathrm{n}=17)$ & 34 \\
& $\mathbf{1 0 0}$ & $\mathbf{8 0 0}$ & $\mathbf{4 1 5} \pm \mathbf{9 1 . 6 1}(\mathbf{n}=\mathbf{1 0})$ & $\mathbf{6 9 . 8}$ \\
Profundidad $(\mathrm{cm})$ & 100 & 1050 & $332.35 \pm 306.66(\mathrm{n}=17)$ & 92.27 \\
& $\mathbf{2 7}$ & $\mathbf{9 4 . 5}$ & $\mathbf{6 0 . 2 7} \pm \mathbf{2 2 . 7 6}(\mathbf{n}=\mathbf{1 0})$ & $\mathbf{3 7 . 8}$ \\
\hline
\end{tabular}

* $\mathrm{n}=$ número de subestaciones. En OD (\%) solo 9 subestaciones evaluadas.

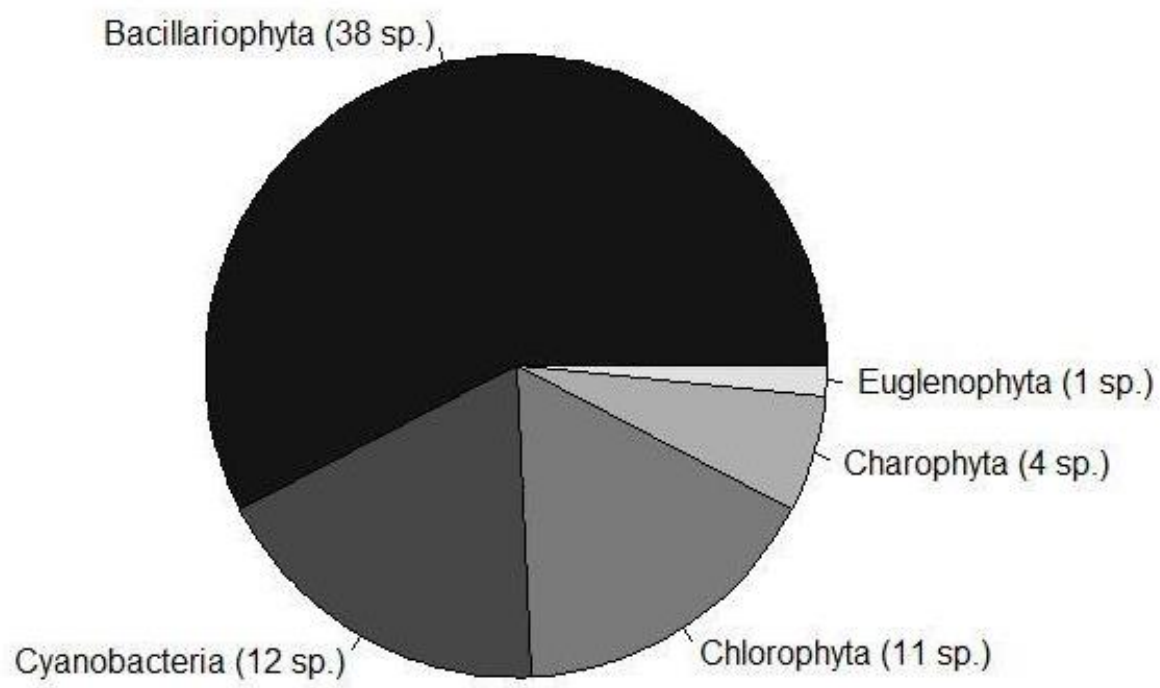

Figura 2. Número de especies por phyla. 


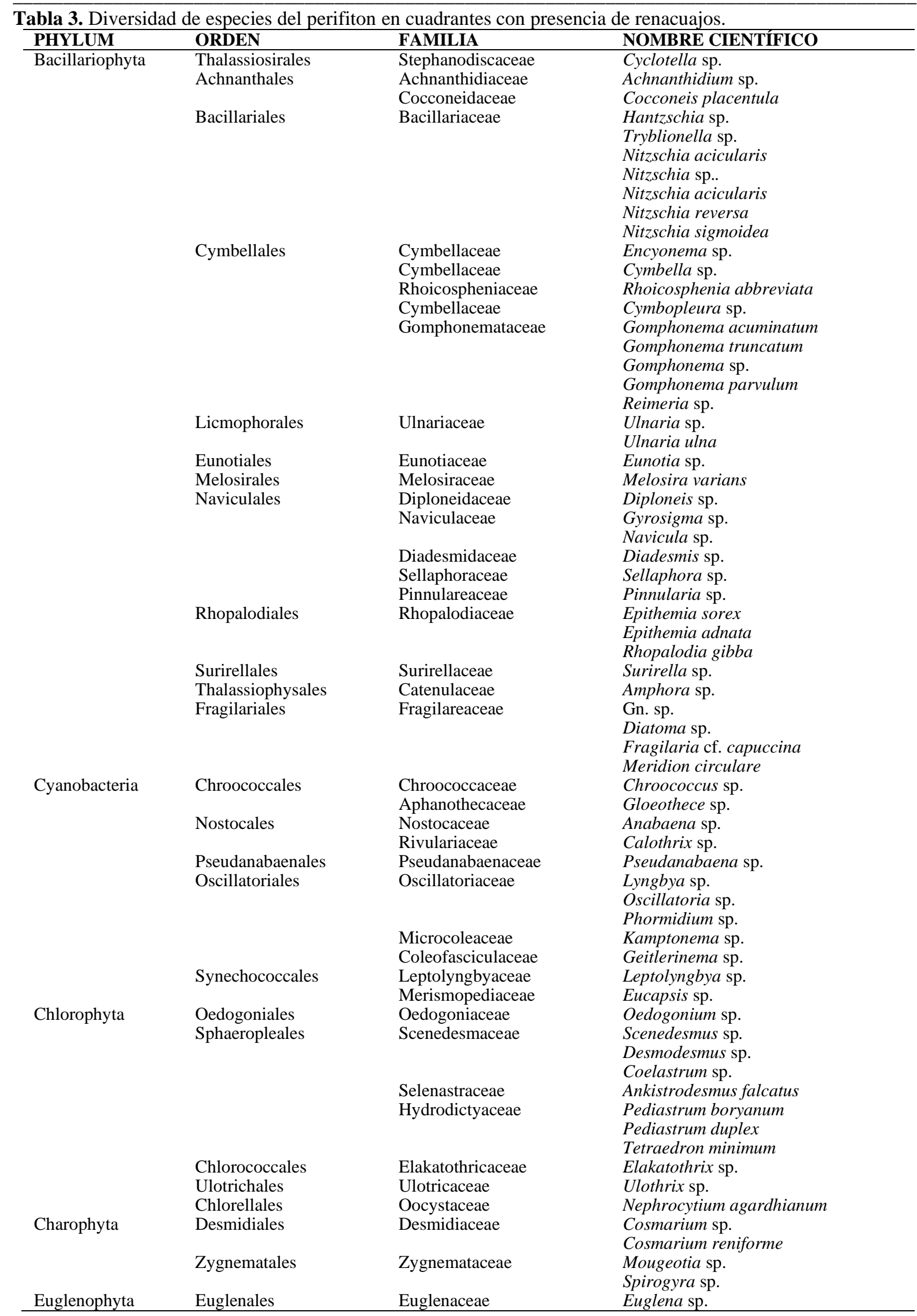


${ }^{1}$ Asociación Grupo RANA. San Martin de Porres 15112 / Lima / Perú.

${ }^{2}$ Denver Zoological Foundation. Denver / Colorado / USA.

${ }^{3}$ Museo de Historia Natural / Universidad Nacional Mayor de San Marcos. Av. Arenales 1256 / Jesús María. Ap. 14-0434, Lima / Perú.

${ }^{4}$ Autor de correspondencia. lcastillo.rana@gmail.com.

${ }^{5}$ Facultad de Medicina Veterinaria y Zootecnia / Universidad Peruana Cayetano Heredia. Lima / Perú. 